\title{
The Real Cause of Differential Aging in the Twin "Paradox"
}

\author{
Gordon Liu \\ 38 Carolyn Crescent, Melbourne, VIC 3083, Australia \\ E-mail: gordonliu168@gmail.com \\ 8 February 2022
}

\begin{abstract}
Physicists have employed various approaches to solve the "paradox" and offered a consistent conclusion that the traveller twin is younger than the homebody twin. However, some authors attribute the cause of differential aging to acceleration, while others believe that acceleration should not be regarded as the source of differential aging, but frame switch is. So far there is no agreement on this issue. Actually, acceleration and frame switch should not be regarded as the source of differential aging. The author will introduce a thought experiment in which two very long spacecraft are moving in opposite directions at a speed close to the speed of light to discuss the real cause of differential aging. The author found that the real cause of differential aging in the twin "paradox" is because of the asymmetry of destination choice. People always choose their destinations in the universe rather than in spacecraft. The destinations are always stationary relative to the homebody twin and moving relative to the traveller twin. Once we choose a destination in a reference frame, the distance is the proper length in this reference frame, but the distance will contract in another moving reference frame, thus the recording time will be shorter.
\end{abstract}

Keywords:Twin paradox, Relativity, Space-time, Time dilation, Differential aging

\section{Introduction}

There are thousands of articles discussing and explaining the twin "paradox" since Paul Langevin first posed the twin "paradox" in 1911. Langevin described a scenario in which the traveler twin leaves the Earth for a distant star at a speed close to the speed of light and returns in the same manner being younger than the homebody twin on the Earth; On the other hand, according to the relativity of motion, from the traveler twin's perspective, the homebody twin leaves the spacecraft for a long distance travel and returns should being younger than the traveler twin. Who is younger? This is so called the "twin paradox".

The physicists have employed various approaches, such as space-time diagram [1][2], special relativity [3]-[10], acceleration and gravity calculus [11]-[20], radar time [21], circular [22], and Doppler effect [23]-[24], etc., to solve the "paradox" and offered a consistent conclusion that the traveler twin is younger than the homebody twin on the 
Earth. However, some authors attribute the cause of differential aging to acceleration $[11,12,14,15,17,20,21]$, while others believe that accelerations should not be regarded as the source of differential aging, but frame switch is $[1,9,18]$. So far there is no agreement on this issue.

As John B Kogut says, "we never have to consider acceleration" (p.35) [1]. One imagines the triplets that, one stays on Earth, other two twins, one outward-going and one inward-coming, who pass each other and synchronize their clocks at the point corresponding to "turnaround" of a single traveler twin. In this version, physical acceleration of the traveler twin plays no direct role. Actually, the frame switch also should not be regarded as the cause of differential aging. As we will discuss below, the homebody twin can send an assistant to the distant star and synchronize their clocks in advance, then measure the time of the single trip of the traveler twin. When the traveler twin reaches the star, the traveler twin and the assistant of the homebody twin will send signals each other to notice the time recorded by their own clocks. This can avoid the effect of acceleration and frame switch, but the differential aging still arises. The traveler twin is younger than the homebody twin. Therefore, we should not attribute the cause of differential aging to acceleration and frame switch.

In view of above issues, the previous explanations were often criticized [25, 26]. Clearly explaining the twin "paradox" is not only to solve the "paradox", but also to deeply understand the relativity of time and space and apply it correctly to quantum physics [27] and spaceflight. In this paper, the author will introduce a thought experiment to show how the differential aging occurs, explain what is the real cause of differential aging, discuss when the age difference is accumulated, and emphasize that the relativity of space-time between the twins still holds.

\section{Two long spacecrafts are moving in the opposite directions at a speed close to the speed of light}

We consider a thought experiment that the two long spacecrafts $A B$ and $A^{\prime} B^{\prime}$ are moving in the opposite directions at a speed close to the speed of light $\mathrm{v}$ in the inertial reference frame E. Two identical spacecrafts are equipped with the same light clocks. Similar to the examples introduced in most textbooks, the light is reflected between the mirrors on the top and bottom of the train. Laser sensor devices are installed on each prow and stern of the spacecraft. When two laser sensor devices are close, the devices can record and sound alarms. The two long spacecrafts $A B$ and $A^{\prime} B^{\prime}$ have same proper length $\mathrm{L}$. As shown in fig. 1, the two spacecrafts pass the point $O$ in the opposite directions in the inertial reference frame $\mathrm{E}$ with same uniform speed $\mathrm{v}$, but on the different close parallel trajectories. When the prows $A$ and $A^{\prime}$ of the spacecrafts pass the point $\mathrm{O}$ (called the event PP), the observers of two spacecrafts start to record their time and synchronize all own clocks in their spacecrafts using Einstein's method.

Now some observers stand in the reference frame E to observe the two spacecrafts and calculate the time when the prows and sterns of two spacecrafts are aligned. The 


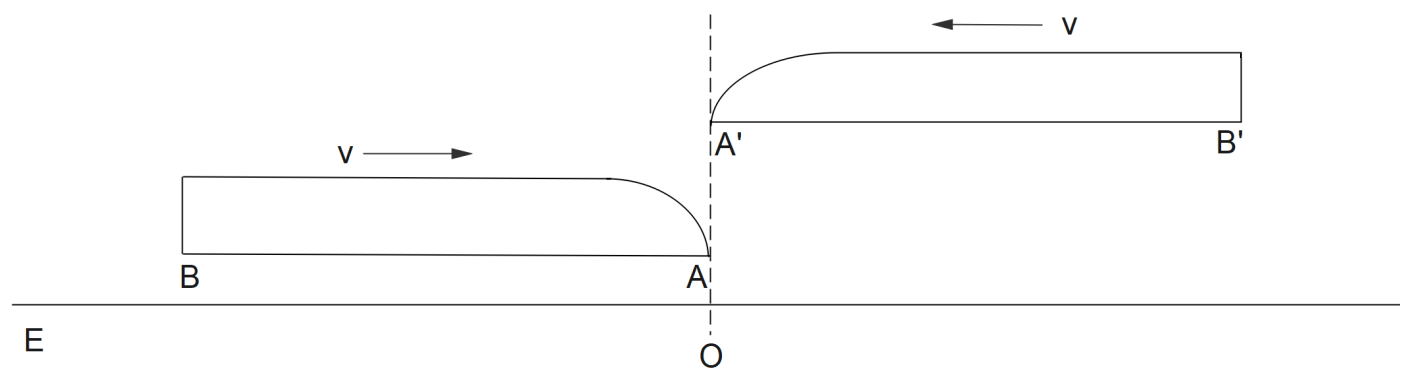

Figure 1. Two spacecrafts $A B$ and $A^{\prime} B^{\prime}$ move in the opposite direction with same speed $\mathrm{v}$ in reference frame $\mathrm{E}$.

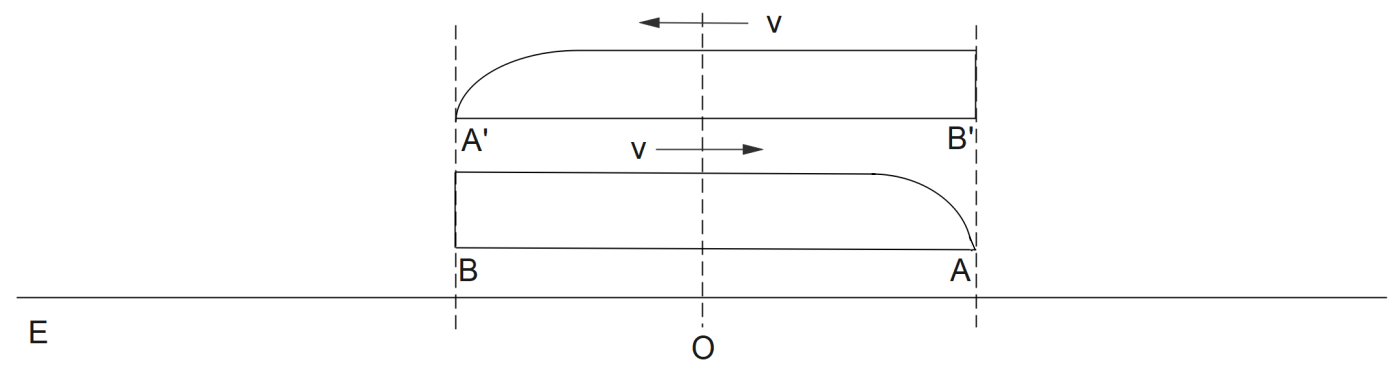

Figure 2. The prows and sterns of the two spacecrafts are aligned at a certain moment.

event in which the prow of one spacecraft reaches the stern of another spacecraft is called event "PS". The event in which the stern of one spacecraft passes the prow of another spacecraft is called event "SP". Of cause, they can align at a moment because they have same length as shown in fig. 2. This means that the two events $A^{\prime} B$ alignment (PS or $\mathrm{SP}$ ) and $B^{\prime} A$ alignment (SP or PS) can occur simultaneously in the reference frame E.

In the reference frame $\mathrm{E}$, the spacecraft $A B$ and $A^{\prime} B^{\prime}$ have same length,

$$
\overline{A B}=\overline{A^{\prime} B^{\prime}}=L \sqrt{1-v^{2} / c^{2}} .
$$

The prows and sterns of the two spacecrafts are aligned at the time

$$
T_{E}=\frac{\overline{A B}}{2} \cdot \frac{1}{v}=\frac{\overline{A^{\prime} B^{\prime}}}{2} \cdot \frac{1}{v}=\frac{L \sqrt{1-v^{2} / c^{2}}}{2 v} .
$$

At this moment, the laser sensor devices on both ends of the two spacecrafts will sound alarms at the same time.

Next we calculate what the observers in the spacecraft $A B$ observe (Fig. 3). They found the spacecraft $A^{\prime} B^{\prime}$ passed by the spacecraft $A B$ with the speed $\mathrm{u}$,

$$
u=\frac{(-v)-v}{1-(-v) v / c^{2}}=-\frac{2 v}{1+v^{2} / c^{2}} .
$$

Now there are two scenarios to observe the spacecraft $A^{\prime} B^{\prime}$. First, the prow $A^{\prime}$ of the spacecraft $A^{\prime} B^{\prime}$ moves from the prow $A$ (event PP) to the stern $B$ (event PS) of the spacecraft $A B$. We call this scenario as "PP-PS" (Prow Prow then Prow Stern); Second, the observers record the time from the moment of the prow $A^{\prime}$ passing the prow 


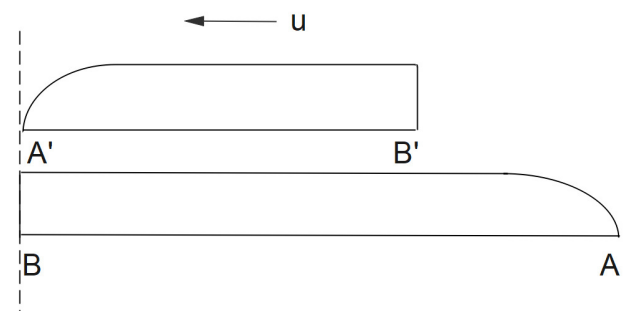

Figure 3. The prow $A^{\prime}$ is reaching the stern $B$ after passing the prow $A$.

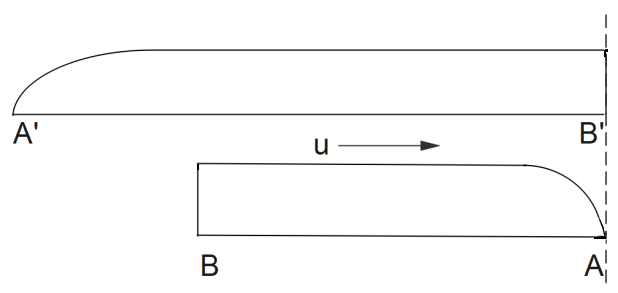

Figure 4. The prow $A$ is reaching the stern $B^{\prime}$ after passing the prow $A^{\prime}$.

$A$ (event PP) to the moment of the stern $B^{\prime}$ passing the prow $A$ (event $\mathrm{SP}$ ). We call this scenario as "PP-SP" (Prow Prow then Stern Prow). In the first scenario, the moving distance is $\mathrm{L}$. The time is

$$
T_{P P-P S}=L / u \text {. }
$$

While in the second scenario, the moving distance is

$$
\ell=L \sqrt{1-u^{2} / c^{2}}
$$

thus the time is

$$
T_{P P-S P}=\frac{\ell}{u}=\frac{L \sqrt{1-u^{2} / c^{2}}}{u} .
$$

From the formulas (Eq. 4) and (Eq. 6), we know that the time $T_{P P-P S}$ in the scenario PP-PS is longer than the time $T_{P P-S P}$ in the scenario PP-SP. Actually, in the scenario PP-PS, before the prow $A^{\prime}$ reaches the stern $B$, the stern $B^{\prime}$ has already passed the prow $A$, while in the scenario PP-SP, after the stern $B^{\prime}$ has passed the prow $A$, the prow $A^{\prime}$ has not reached the stern $B$. This means that, in the spacecraft $A B$, the two events of the prow $A^{\prime}$ reaching the stern $B$ (event PS) and the stern $B^{\prime}$ passing the prow $A$ (event $\mathrm{SP}$ ) are not simultaneous anymore.

The same scenarios will happen in the spacecraft $A^{\prime} B^{\prime}$ (Fig. 4) when observing the spacecraft $A B$, because the motions of the spacecraft $A B$ and the spacecraft $A^{\prime} B^{\prime}$ are exactly symmetric.

Doing the same calculations, we know that the time $T_{P P-P S}^{\prime}$ is longer than the Time $T_{P P-S P}^{\prime}$ (Adding an apostrophe after $\mathrm{T}$ denotes the time is recorded in the spacecraft $\left.A^{\prime} B^{\prime}\right)$.

From the above discussion, we find that the recording time in the scenario PP-PS is always longer than that in the scenario PP-SP. The differential has to do with which 
scenario we choose to measure or which final recording point in time we choose after the two prows of the spacecraft have passed. Note that the "recording point in time" or "recording time point" is the position and time of an event used to record a process. If choosing the recording point in time $A^{\prime} B$ as the final recording point in time, at this moment, the prow $A^{\prime}$ reaches the stern $B$, namely the event PS occurs, thus the clocks in the spacecraft $A B$ record a longer time than the ones in the spacecraft $A^{\prime} B^{\prime}$. That is because the observers in the spacecraft $A B$ are in the scenario PP-PS, while the observers in the spacecraft $A^{\prime} B^{\prime}$ are in the scenario PP-SP. We say the time is dilated in the spacecraft $A^{\prime} B^{\prime}$. In turn, if choosing the recording point in time $A B^{\prime}$ as the final recording point in time, at this moment, the prow A reaches the stern $B^{\prime}$, namely the event PS occurs. For a similar reason, the clocks in the spacecraft $A^{\prime} B^{\prime}$ record a longer time (PP-PS) than the ones in the spacecraft $A B$ (PP-SP). We say the time in the spacecraft $A B$ is dilated.

Now we check the light clocks equipped in both spacecrafts. Just like the description in the textbooks, the observers on the spacecrafts find that the other party's light path from bottom to top and from top to bottom is not perpendicular to the spacecraft, but an oblique line, while the light path is perpendicular to the spacecraft on their own spacecraft. The light clocks in their own spacecraft have no change, but are dilated when observing from the other party's spacecraft.

Therefore, we have the following conclusions: First, the uniform rectilinear motion is relativistic. Second, the inertial reference frames are equal and symmetric. Third, the time and space (length) are relativistic between the inertial reference frames. Fourth, the clocks in the inertial reference frames have no change in mechanism. Fifth, the choice of the final recording point in time (PS or SP) in the two spacecrafts is exactly the opposite, because the scenario is exactly the opposite (PP-PS or PP-SP). The scenario PP-PS in one spacecraft (the final recording point in time is PS) presents another scenario PP-SP (the final recording point in time is SP) in another spacecraft. In other words, a process (two events) presents a different scenario in each frame of reference that moves with each other. Sixth, shorter or longer of the time recorded by the clocks in the two spacecrafts depends on the choice of the final recording point in time, PS or SP, after the first recording point in time (PP) has been chosen.

\section{On the twin "paradox"}

We apply above thought experiment and the conclusions to twin "paradox". Since the differential aging will still occur without acceleration and frame switch, acceleration or frame switch should not be regarded as the real cause of differential aging. There are no harm to imagine the traveler twin's round trip as two single trips back and forth.

In the twin "paradox" scenario, the Earth and the distant star can be considered as an inertial reference frame and the spacecraft is another inertial reference frame. The Earth-star system can be imagined as a spacecraft whose prow is the Earth and its stern is the distant star, while the traveler twin in the spacecraft is just seen as the prow of 
a long spacecraft because the spacecraft is commonly very short comparing with their traveling distance. The homebody twin can send an assistant to the distant star in advance and then they synchronize their clocks. The traveler twin takes off from the Earth and undergoes a short accelerating period, and then starts his/her journey with a fixed speed for a long time cruise to the distant star. Once the traveler twin reaches the star, the traveler twin and the assistant of the homebody twin will send signals each other to notice the time recorded by their own clocks. The homebody twin and his/her assistant observe the traveler twin moves from the Earth to the star. This scenario is PP-PS. Whereas, the traveler twin find the Earth-star system passes by him/her from the Earth (prow) to the star (stern). The final recording point in time is the moment of the star (stern) passing the traveler twin because the star has been chosen in advance as the destination. For traveler twin, the scenario is PP-SP. Therefore, the homebody twin and his/her assistant record a longer time than the traveler twin does. The traveler twin's time has dilated or the traveler twin is younger than the homebody twin. When the traveler twin returns back, the traveler twin's scenario is still PP-SP and the homebody twin's scenario is still PP-PS. Therefore, the traveler twin also is younger than the homebody twin. After traveling forward and returning back, the traveler twin will be younger than the homebody twin.

\section{What is the real cause of differential aging?}

From the above description, we find that the traveler twin travels back and forth in universe is always in the scenario PP-SP, it is impossible to be in the scenario PP-PS. For spaceflight, people always move in the Earth-star reference system and chooses the destinations in the Earth-star reference system, not in the spacecraft. This means that there is asymmetry in the choice of destinations, because the destinations (the stars) are always stationary relative to the homebody twin (the earth) and moving relative to the traveler twin (spacecraft). Therefore the traveler twin can only record a shorter time and is younger than the homebody twin. If the proper length of the spacecraft is very long which is equal to the distance (proper length) from the Earth to the distant star, the traveler twin standing on the prow of the spacecraft and his/her assistant standing on the stern of the spacecraft observe the Earth passing by the traveler twin (the prow of the very long spacecraft) to his/her assistant (the stern of the very long spacecraft), and they choose the moment when the Earth reaches the stern of the very long spacecraft as the final recording point in time. Now the destination (the stern) is stationary relative to the traveler twin and moves relative to the homebody twin (the Earth). In this scenario, the traveler twin is in the scenario PP-PS, while the homebody twin is in the scenario PP-SP. Thus the traveler twin and his/her assistant also can observe that the homebody twin is younger than themselves. Note, in this scenario, before the Earth reaches the stern (the traveler's assistant is staying there) of the very long spacecraft, the traveler twin has passed the distant star.

In regard to the reference frames, during the periods of cruise, the twins both 
are in the inertial reference frame and in equal status. If comparing the light clocks equipped in the each reference system frame, both observers will find the other party's clock is slower because the light path is different. The space-time properties of an inertial reference frame do not change just because it has ever been accelerated. Their space-times are still relativistic. Comparing the time of a process in mutually moving reference frames is to compare the interval between two recording time points of the process. The choice of recording points in time is very important, because it determines the scenario of the process (PP-PS or PP-SP). For example, on the light path of the light clock in the textbooks, if the recording points in time are chosen in the train, the light path is perpendicular to the train, but if choosing on the Earth, the light path is oblique lines. The clocks record shorter or longer time depending on how to choose the recording points in time. Regarding the twin "paradox", the final recording points in time we choose is the moment when the traveler twin reaches the distant star, and the distant star is stationary in the Earth-star system, rather than in the spacecraft. In this way, it makes the traveler twin only in the scenario PP-SP. We see that the reason for this situation is that once we choose a destination in a reference frame, the distance between the starting point and the destination is proper length, but the distance will contract in another moving reference frame, So that the recording time is shorter.

Regarding the role of acceleration, if one wants to calculate the traveling time in more detail, one can consider the acceleration and the differential aging can be expressed in Minkowski space-time as a function of the acceleration and speed [12]-[18], but it does not affect our main conclusion.

\section{Summary}

The real cause of differential aging in the twin "paradox" is because of the asymmetry of destination choice. The destinations (the stars) are always stationary relative to the homebody twin (the earth) and move relative to the traveler twin (spacecraft). Once a destination is chosen in a reference frame, the distance between the starting point and the destination is proper length in the reference frame, but the distance will contract in another moving reference frame, so that the recording time is shorter. Therefore the traveler twin can only record a shorter time and is younger than the homebody twin. For spaceflight, the traveler twin has no choice but to be in the scenario PP-SP, because people always choose the destinations in the universe or in the Earth-star reference frame, but cannot choose their destinations in the spacecraft. In this sense, there is a superior reference frame in our universe. We know that the stars in the universe can form a special reference frame. In this reference frame, any moving clock (or person) will be dilated (or younger ) and any distance traveled will be contracted, but this does not imply that the space-time between the inertial reference frames is not relativistic. Even though we choose the stars or the background of microwave as an absolute rest frame, the special principle of relativity is still correct. The temporal and spatial characteristics of an inertial reference frame will not change just because it has ever been accelerated. 
The time dilation and the length contraction are true for both mutually moving reference frames. The space-time is still relativistic. The time recorded by the clock relies on not only the speed of reference frame, but also the choice of final recording points in time (or the destinations). Generally speaking, comparing the time recorded by the clocks in two mutually moving reference frames is to compare the time records of a process, while the process presents different scenario in each reference frame, thus the time recorded by the clocks is different. However, the clocks in each inertial reference frame have no change in mechanism. The age difference of the twins is accumulated during whole traveling periods including the accelerating, cruising, and decelerating periods.

\section{Acknowledgments}

I am extremely grateful to my wife Rong Zhang for her encouragement and support.

\section{References}

[1] Kogut, John B.: Introduction to Relativity: For Physicists and Astronomers. Academic Press. $35(2012)$.

[2] Muller, R.: The Twin Paradox in Special Relativity. American Journal of Physics, 40 (7), 966976(1972). http://dx.doi.org/10.1119/1.1986722

[3] Romer, R.: Twin Paradox in Special Relativity. American Journal of Physics, 27 (3), 131-135(1959). http://dx.doi.org/10.1119/1.1934783

[4] Bohm, D. and Hiley, B.: Active interpretation of the Lorentz "boosts" as a physical explanation of different time rates. American Journal of Physics, 53 (8), 720-723(1985). http://dx.doi.org/10.1119/1.14300

[5] Debs, T. and Redhead, M.: The twin "paradox" and the conventionality of simultaneity. American Journal of Physics, 64 (4), 384-392(1996). http://dx.doi.org/10.1119/1.18252

[6] Eagle, A.: A note on Dolby ad Gull on radar time and the twin "paradox". American Journal of Physics, 73 (10), 976-979(2005). http://dx.doi.org/10.1119/1.1994855

[7] Price, R. and Gruber, R.: Paradoxical twins and their special relatives. American Journal of Physics, 64 (8), 1006-1008(1996). http://dx.doi.org/10.1119/1.18318

[8] Gruber, R. and Price, R.: Zero time dilation in an accelerating rocket. American Journal of Physics, 65 (10), 979-980(1997). http://dx.doi.org/10.1119/1.18700

[9] Lasky, R.: Time and the Twin Paradox. Scientific American, Special Editions 16 (1s), 2023(February 2006). doi:10.1038/scientificamerican0206-20sp

[10] Frye, R. and Brigham, V.: Paradox of the Twins. American Journal of Physics, 25 (8), 553555(1957). http://dx.doi.org/10.1119/1.1934547

[11] Einstein, A.: The Berlin Years: Writings, 1918-1921 (English Translation of Selected Texts). The Collected Papers of Albert Einstein, 7, Princeton University Press, Princeton, (2002).

[12] Wu, Ta-You and Lee, Y. C.: The Clock Paradox in the Relativity Theory. International Journal of Theoretical Physics, 5 (9), 307-323(1972). https://doi.org/10.1007/BF00678222

[13] Perrin, R.: Twin paradox: A complete treatment from the point of view of each twin. American Journal of Physics, 47 (4), 317-319(1979). http://dx.doi.org/10.1119/1.11835

[14] Iorio, L.: An Analytical Treatment of the Clock Paradox in the Framework of the Special and General Theories of Relativity. Found. Phys. Lett. 18 (3), 1-19(2005). DOI.org/10.1007/s10702005-2466-8

[15] Grøn, Ø.: The twin paradox and the principle of relativity. Physica Scripta, 87 (3), 8-21(2013). DOI: $10.1088 / 0031-8949 / 87 / 03 / 035004$ 
[16] Good, R.: Uniformly accelerated reference frame and twin paradox. American Journal of Physics, 50 (3), 232-238(1982). http://dx.doi.org/10.1119/1.12862

[17] Nikolić, H.: The role of acceleration and locality in the twin paradox. Found. Phys. Lett. 13 (12), 595-601(2000). https://doi.org/10.1023/A:1007866532278

[18] Minguzzi, E.: Differential aging from acceleration: An explicit formula. American Journal of Physics, 73 (9), 876-880(2005). http://dx.doi.org/10.1119/1.1924490

[19] Styer, D.: How do two moving clocks fall out of sync? A tale of trucks, threads, and twins. American Journal of Physics, 75 (9), 805-814(2007). http://dx.doi.org/10.1119/1.2733691

[20] Gamboa, J.: F. Mendez, M.B. Paranjape, The "twin paradox": the role of acceleration. Canadian Journal of Physics, 97 (10), 1-17(2019). https://doi.org/10.1139/cjp-2018-0788

[21] Dolby, C. and Gull, S.: On radar time and the twin "paradox". American Journal of Physics, 69 (12), 1257-1261(2001). http://dx.doi.org/10.1119/1.1407254

[22] Cranor, M., Heider, E. and Price, R.: A circular twin paradox. American Journal of Physics, 68 (11), 1016-1020(2000). http://dx.doi.org/10.1119/1.1286313

[23] Blatter, H. and Greber, T.: Aberration and Doppler shift: An uncommon way to relativity. American Journal of Physics, 56 (4), 333-338(1988). http://dx.doi.org/10.1119/1.15633

[24] Müller, T., King, A. and Adis, D.: A trip to the end of the universe and the twin "paradox". American Journal of Physics, 76 (4), 360-373(2008). http://dx.doi.org/10.1119/1.2830528

[25] Wang, Ling Jun: Symmetrical Experiments to Test the Clock Paradox. Physics and Modern Topics in Mechanical and Electrical Engineering, Ed., Nikos Mastorakis, World Scientific and Engineering Society Press, ISBN: 960-8052-10-6, 45-50(1999). doi:10.1.1.510.5842

[26] Kak, S.: Moving Observers in an Isotropic Universe. International Journal of Theoretical Physics, 46 (1), 1424-1430(2007). https://doi.org/10.1007/s10773-006-9281-2

[27] Loriani, S.: A. Friedrich, etc. Interference of clocks: A quantum twin paradox. Sci. Adv. 5 (10), eaax8966(2019). doi: 10.1126/sciadv.aax8966 